\title{
Development of a Novel Penetration-Enhancing Agent for Hair Products
}

\author{
Teruaki Nagasawa $^{1}$, Hiroyuki Suzuki ${ }^{1}$, Masako Koyama ${ }^{1}$, Toshiko Sato ${ }^{1}$, Kimio Kawamura ${ }^{1}$, \\ Yoko Yamaguchi ${ }^{1,2}$
}

${ }^{1}$ NANOEGG ${ }^{\circledR}$ Research Laboratories, Inc., Kawasaki, Japan; ${ }^{2}$ Institute of Medical Science, St. Marianna University School of Medicine, Kawasaki, Japan.

Email: teruaki-nagasawa@nanoegg.co.jp

Received November $15^{\text {th }}, 2012$; revised December $20^{\text {th }}, 2012$; accepted December $30^{\text {th }}, 2012$

\begin{abstract}
HAIRCARECUBE $^{\mathrm{TM}}(\mathrm{HCC})$ is a new additive for hair products that allows the active ingredient to penetrate into the hair. During the course of percutaneous absorption studies, we discovered that lyotropic liquid crystal promotes the skin's absorptiveness to medicines. We conducted a study to determine whether or not lyotropic liquid crystal could be applied as a penetration-enhancing agent in hair, and as a result we have succeeded in developing HCC. In place of hair dye, we used fluorescein-HCC to evaluate hair permeability, and strong fluorescence was observed as deep as the core of the hair. Moreover, the strength of the fluorescence was dependent on HCC concentration. This result did not change under acidic or basic conditions. In addition, the same trends were observed when using an oxidative hair dye. Furthermore, when HCC was used together with hydrolyzed keratin, which repairs hair damage, a stronger restorative effect was observed. These results confirmed that $\mathrm{HCC}$ has the effect of promoting the permeation of pigments and other active ingredients into hair. HCC is expected to be very useful as an additive for developing functional cosmetic hair products.
\end{abstract}

Keywords: Permeation; Hair Care; Hair Dye; Hair Repair; HAIRCARECUBE ${ }^{\mathrm{TM}}$ (HCC)

\section{Introduction}

Hair care needs have diversified over the years. In recent times, treatments to combat the graying and thinning of hair with age have been of particular interest, and the availability of hair care products and hair dyes has increased accordingly. In addition, various commercial products have been developed with the help of new technologies to beautify the hair in numerous ways, such as polishing, coloring, or perming the hair $[1,2]$. Thus, the number of hair care products has been increasing dramatically, and consumers are on the lookout for simple but highly effective products. Efficacy is dependent on the successful delivery of active ingredients to the desired location in the hair, namely through the cuticle and into the cortex.

Just as a drug delivery system (DDS) is essential for drug development in the pharmaceutical industry, DDS technology is also applicable to the development of functional hair care products. In the pharmaceutical industry, DDS technology is essential for ensuring the successful arrival of the drug at the desired location in the body. For example, liposomes [3], a typical example of a DDS, are already in clinical use, and polymetric micelles, currently in the clinical trial stage, are following close behind $[4,5]$. Recently, our group developed a novel transdermal delivery system (TDS) with a special liquid crystal structure that allows drugs to penetrate the skin through the intercellular lipids in the stratum corneum [6-10].

In order to meet consumer demand, it is necessary to introduce this DDS technology to the hair care industry and enhance the permeability of the hair to desirable active ingredients. Up until now, there has been a strong focus on macroscopic properties, and hair penetrationenhancing products have been created using organic solvents, oil-based mediums, and surfactants - but there are concerns that these methods may actually disrupt the structure of the hair and cause serious damage [11]. By contrast, we focused on the nanostructure of the hair and targeted the cell-membrane complex (CMC) [12], a layer separating the cuticle surrounding the hair from the cortex at the core of the hair. With the results of our TDS research in mind, we succeeded in creating HAIRCARE$\mathrm{CUBE}^{\mathrm{TM}}$ (HCC), a novel penetration aid for hair care products.

In this paper, we introduce the properties of $\mathrm{HCC}$ and investigate the impact of $\mathrm{HCC}$ on the permeability of hair 
to active ingredients such as pigments and damage-repair agents. In order to directly test the degree of penetration, we used fluorescein as a model pigment, and we evaluated the impact of HCC on the level of fluorescence inside the hair. These experiments were carried out using damaged hair rather than healthy hair since it is believed that the damaged state has become more common. In addition to the damage incurred every day as a result of UV ray exposure, the friction from hair brushes, and the heat from hair dryers, the level of damage has skyrocketed since the advent of bleaching, dying, and perming the hair in the 1990s. We investigated whether or not HCC could improve the penetration of hair repair agents as well as dyes. As keratin is known to increase the strength of hair fibers, we conducted experiments using hydrolyzed low molecular weight keratin, which alone has little effect on hair fiber strength [13-15]. We determined the level of penetration indirectly by comparing the strengths of the hair fibers after different treatments.

\section{Materials and Methods}

\subsection{HAIRCARECUBE ${ }^{\mathrm{TM}}$ (HCC)}

$\mathrm{HCC}$ is novel penetration aid with a unique mechanism that allows active ingredients to permeate the CMC. The lyotropic liquid crystal structure of HCC is created by mixing the proper amounts of four ingredients together in series: glycerin, water, oil, and a surfactant. When dissolved in water, HCC dissolves to transparency due to its specific nano-scale structure; however, when the ingredients that compose HCC are dissolved in water together at equal concentrations, the mixture is cloudy and precipitates form [16].

Because pure HCC is a thick, semi-transparent gel resembling Vaseline ${ }^{\mathrm{TM}}$, HCC should be diluted before use in hair products, and it is usually present at only a few percent. Due to its solubility and stability in water, it is thought that $\mathrm{HCC}$ can be easily blended into various hair products without threatening the stability of the merchandise.

\subsection{Fluorescein Study}

In order to effectively assess the capacity of HCC to increase the permeability of the hair to pigment, the easily visualizable and quantifiable fluorescein was used as a model pigment. Bleached hair was prepared by immersing commercially available human hair in a 1:1 mixture of aqueous $10 \%$ hydrogen peroxide and 5\% ammonia (Wako Pure Chemical Industries, Ltd., Japan) and processing for $30 \mathrm{~min}$ at $37^{\circ} \mathrm{C}$. Fluorescein (Sigma-Aldrich ${ }^{\circledR}$ Co., Ltd., Japan) was dissolved to a final concentration of $0.1 \%$ in aqueous solutions with fixed concentrations of $\mathrm{HCC}(0 \%, 0.1 \%, 0.5 \%, 1 \%, 2 \%$, and $5 \%)$. For the pur- poses of comparison, fluorescein was also added to two control solutions: 1) a simple mixture of the four ingredients composing $\mathrm{HCC}$, each present at a $2 \%$ concentration; and 2) a commercially available product. After processing again for $30 \mathrm{~min}$ at $37^{\circ} \mathrm{C}$, the hair was rinsed to remove any residual pigment and dried. The dried hair was embedded in Tissue-Tek O.C.T. Compound (Sakura Finetek Japan Co., Ltd., Japan), frozen, sectioned at $5 \mu \mathrm{m}$, and mounted on glass slides. The sections were observed under a fluorescent microscope (Biozero Fluorescence microscope BZ-8100, KEYENCE, Japan) and analyzed using the included software (BZ analyzer, KEYENCE, Japan).

To assess the efficacy of HCC under acidic and alkaline conditions, fluorescein was dissolved in aqueous solutions of $0.5 \% \mathrm{HCC}$ with varying $\mathrm{pH}$. Hair was processed as above, and these cross-sections were also observed under the fluorescent microscope and analyzed as above.

\subsection{Oxidizing Dye Study}

An experiment using an oxidizing dye was conducted in a similar fashion to that of the experiment described above using the model pigment fluorescein. Bleached hair was soaked in solutions containing oxidizing dye and $\mathrm{HCC}$ at various concentrations $(0 \%, 2 \%$, and $5 \%)$, allowed to process for $30 \mathrm{~min}$ at $37^{\circ} \mathrm{C}$, rinsed, and dried. The hair was then embedded in the same O.C.T. compound as above, frozen, sectioned at $15 \mu \mathrm{m}$, and mounted on glass slides. The slides were observed under an optical microscope (Biozero Fluorescence microscope BZ8100, KEYENCE, Japan), and image analysis was performed using image processing software (Image J Windows).

\subsection{Keratin Study}

The level of keratin penetration was determined indirectly by measuring the strength of hair fibers after various treatments. Hair processed with bleach was soaked in aqueous solutions containing 5\% hydrolyzed keratin (molecular weight of approximately $400 \mathrm{~g} / \mathrm{mol}$ ) and various concentrations of $\operatorname{HCC}(0 \%, 2 \%, 5 \%$, and $10 \%)$. For the purposes of comparison, two control solutions were also prepared as before: 1) a simple mixture of the four ingredients composing HCC, each present at a $2 \%$ concentration; and 2) a commercially available product. After allowing the hair to process for $30 \mathrm{~min}$ at $37^{\circ} \mathrm{C}$, the hair was rinsed and dried. These hair samples, along with samples of untreated healthy hair and bleached hair, were tested for resistance to breaking under the strain of weight as an indicator of hair fiber strength and therefore an indicator of the efficacy of the keratin treatment. The data was normalized such that a unit of 1 corresponds to 
the amount of weight required to break a single fiber of healthy hair.

\section{Results and Discussion}

\subsection{Relative Efficacy of HCC}

The fluorescent photomicrographs of hair cross-sections shown in Figure 1 (top) revealed that HCC increased the capacity of the fluorescein model pigment to penetrate the hair fibers. When compared to the $0 \%$ HCC group, it was clear that the $2 \%$ HCC group displayed not only stronger fluorescence around the circumference of the hair but also greater overall fluorescence. Moreover, the 5\% HCC group showed strong fluorescence throughout the entire cross-section, indicating that the fluorescein pigment permeated throughout the entire hair fiber.

As noted earlier, a simple mixture of the ingredients that compose HCC should not have the same effect as properly formulated HCC. As expected, the mixture of the four HCC components, each present at a concentration of $2 \%$, showed little effect when compared to $2 \%$ $\mathrm{HCC}$ or $5 \%$ HCC. In fact, the pattern of fluorescence in this group was very similar to that of the commercial product group. It is suspected that this small effect was due to the presence of a surfactant among the HCC components.

The graph in Figure 1 (bottom) shows a graphical representation of the fluorescence shown in the photomicrographs; the intensity along the diameter of each cross-section is displayed beginning from the cuticle on one side, passing through the cortex into the medulla, and continuing across to the cuticle on the other side. As compared to the commercial product, remarkably high intensity fluorescence was observed in the HCC groups throughout the cuticle and cortex layers, which was consistent with the results shown in the photomicrographs.

Figure 2 shows a numerical representation of the fluorescence intensity of fluorescein in the hair fibers after various treatments. It was determined that the increases in fluorescence in all HCC groups $(0.1 \%$ HCC and higher) were statistically significant when compared to the simple aqueous fluorescein treatment group ( $0 \% \mathrm{HCC})$. In fact, the level of fluorescein permeation in the hair increased in an HCC concentration-dependent manner. Treatments containing $2 \%$ or more HCC displayed fluorescence significantly greater than that of the simple mixture of the HCC components or the commercial product.

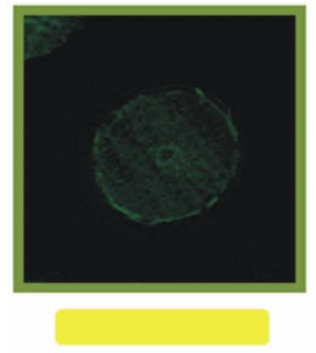

$0 \% \mathrm{HCC}$

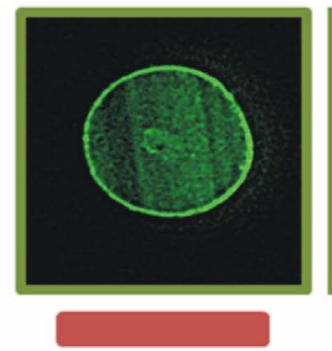

$2 \% \mathrm{HCC}$

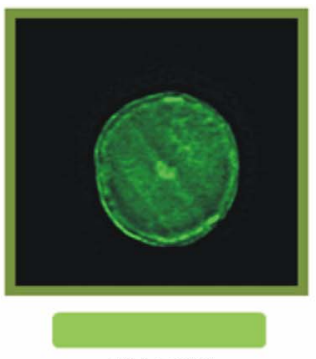

$5 \% \mathrm{HCC}$

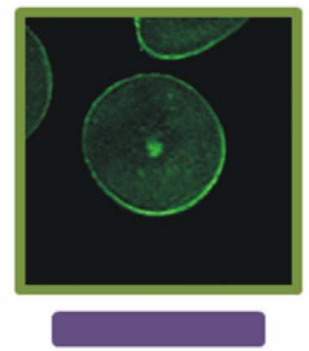

$2 \% \mathrm{HCC}$

Components
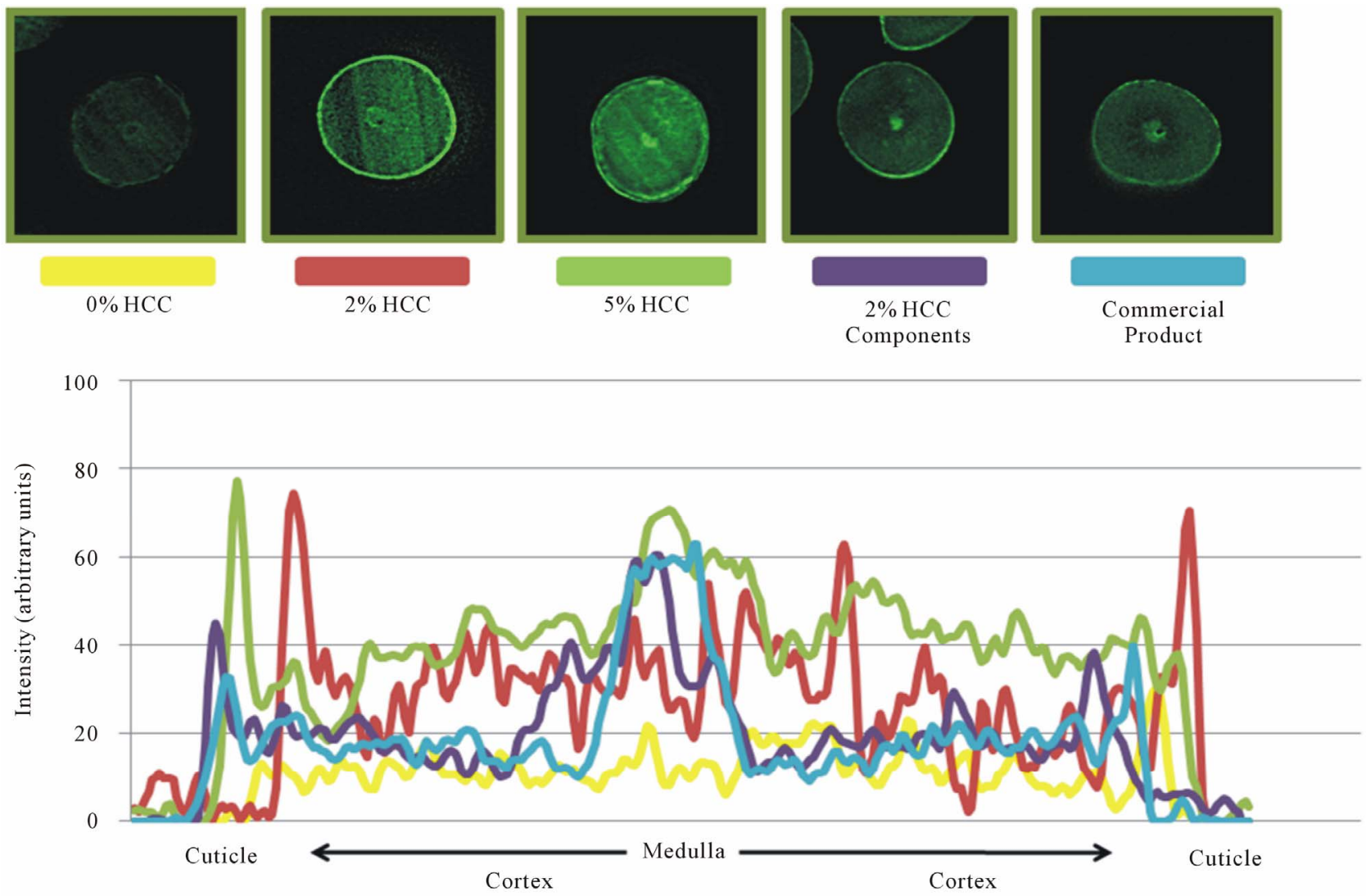

Figure 1. Penetration of fluorescein model pigment into hair fibers. Representative photomicrographs (top) of hair crosssections visualized under a fluorescent microscope and a plot (bottom) displaying the quantified levels of fluorescence along the diameters of the circular cross-sections. 


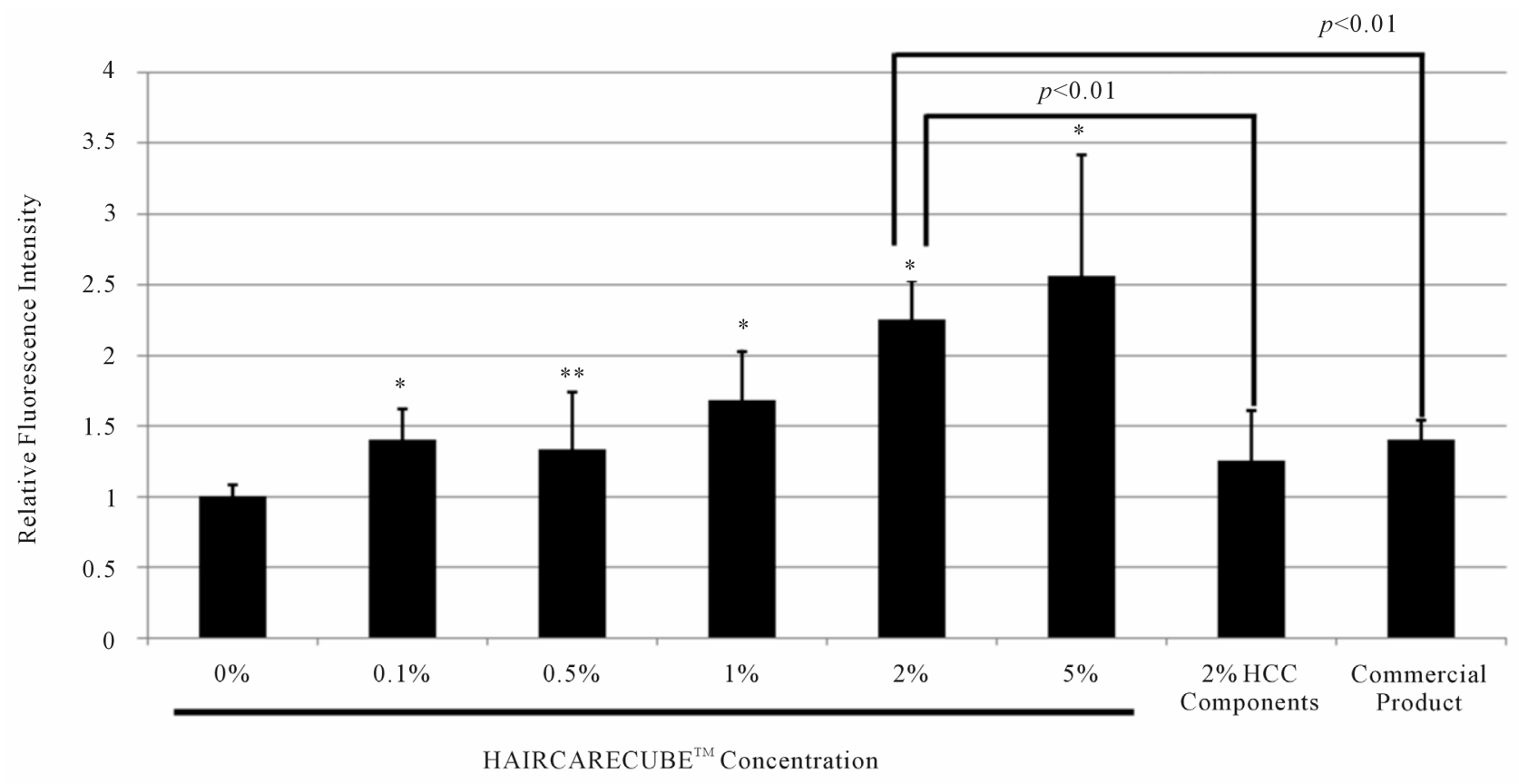

Figure 2. Relative amount of penetration of fluorescein model pigment into hair fibers with respect to HCC concentration. Asterisks denote significant differences from the $0 \%$ HCC control: ${ }^{*} p<0.01 ;{ }^{* *} p<0.05$.

\section{2. pH-Insensitive Stability of HCC}

Neither acidity nor alkalinity adversely affected the performance of HCC. The representative photomicrographs in Figure 3 illustrate that even under acidic $(\mathrm{pH} 2.5)$ or alkaline ( $\mathrm{pH}$ 10.9) conditions, the fluorescence inside the hair fibers appeared similar to that of the fibers treated with HCC under neutral (pH 7.0) conditions, indicating that the effectiveness of HCC was unchanged by the variation in $\mathrm{pH}$.

This $\mathrm{pH}$-insensitive stability is thought to be due to the special composition of HCC. Firstly, HCC is composed of four ingredients, each of which is very stable even under acidic or alkaline conditions: water, oil, glycerin, and a surfactant. Secondly, HCC exists in the form of a liquid crystal, which is an especially stable type of structure in aqueous environments. As the composition of commercial hair products is widely varied, this stability is considerably valuable in the hair care industry.

\subsection{Efficacy of HCC with Oxidative Dyes}

The degree of penetration of the oxidative dye increased with the addition of HCC in a concentration-dependent manner, exhibiting the same trend as was the case with the fluorescein model pigment. The graph in Figure 4 shows that increasing the concentration of HCC from $0 \%$ to merely $2 \%$ yielded a statistically significant increase in the amount of dye that penetrated the hair fibers. This study served to confirm that HCC can be added to oxidative dyes to enhance penetration, thereby enhancing the

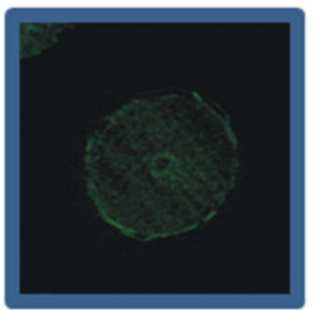

Water

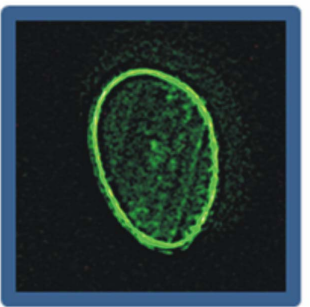

$0.5 \% \mathrm{HCC}, \mathrm{pH} 2.5$

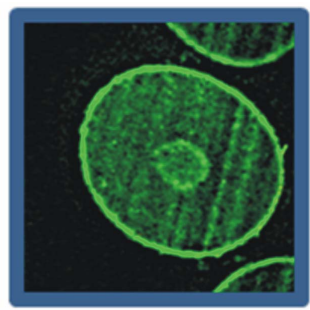

$0.5 \% \mathrm{HCC}, \mathrm{pH} 7.0$

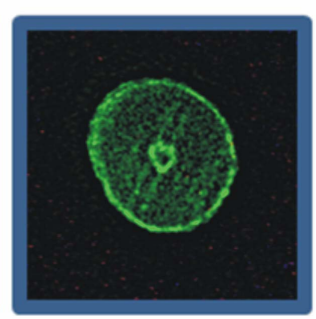

$0.5 \% \mathrm{HCC}, \mathrm{pH} 10.9$
Figure 3. Photomicrographs showing level of HCC-enhanced penetration of fluorescein model pigment with respect to pH of treatment.

effectiveness of the hair dye. This remarkable increase in the amount and depth of penetration implies that the addition of HCC to hair dyes would markedly improve color retention.

\subsection{Efficacy of HCC with Hydrolyzed Keratin}

The effectiveness of hydrolyzed keratin in repairing damage and restoring strength to hair was significantly 


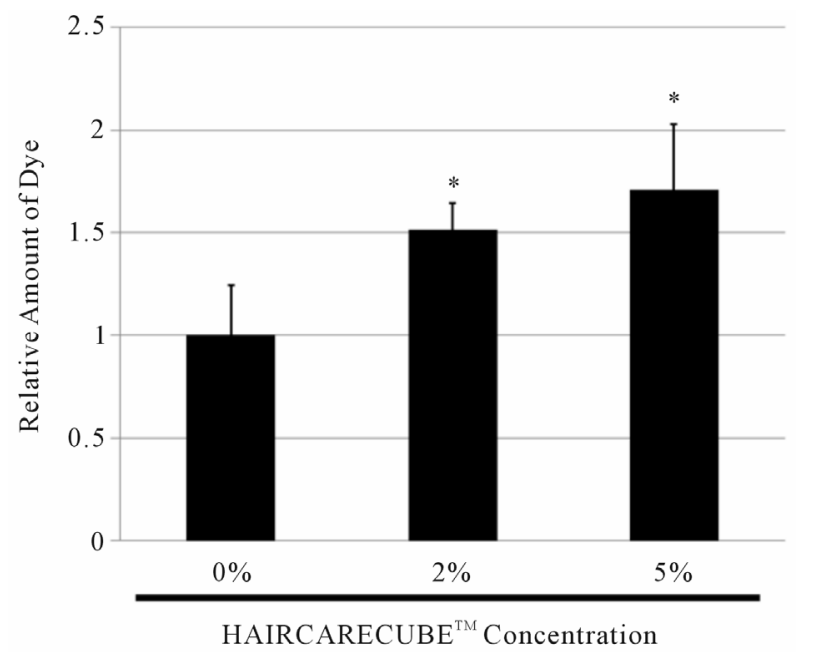

Figure 4. Relative amount of penetration of oxidative dye into hair fibers with respect to HCC concentration. Asterisks denote significant differences from the $0 \%$ HCC control: ${ }^{*} p<0.01$.

amplified by the addition of HCC. The graph in Figure 5 shows the relative strengths of the tested hair fibers, where strength was equated to the amount of weight required to break a strand of hair. The data was normalized such that a value of 1 represents the weight required to break a single strand of healthy hair, and therefore a value of 1 represents the strength of a healthy strand of hair. At a concentration of $2 \%$ or greater, the addition of
HCC enhanced the reparative effect of hydrolyzed keratin beyond that exhibited by the commercial product. At a concentration of $5 \%$ or greater, the addition of HCC yielded a significant difference in strength compared to the hydrolyzed keratin alone $(0 \%$ HCC) treatment group. As was the case with fluorescein, the addition of a simple combination of the ingredients that compose HCC did not have any effect, and the result was similar to that of the hydrolyzed keratin alone $(0 \% \mathrm{HCC})$ treatment group. This study demonstrated that HCC can be effective in hair repair products as well as in hair dyes.

\section{Conclusions}

The demand for hair dyes and hair repair products is on the rise, and one should not underestimate the significance of a simple additive capable of increasing the effectiveness of the dyes and reparative agents.

We adapted the research we conducted on transdermal drug delivery systems, and we created HAIRCARE$\mathrm{CUBE}^{\mathrm{TM}}$ (HCC). In this study, we established that HCC possesses the capacity to increase the penetration of pigments and other active ingredients into the core of the hair fibers. Even at low concentrations, HCC is able to significantly increase the effectiveness of hair products, and HCC is stable even under acidic or basic conditions.

In addition, the liquid crystal structure of $\mathrm{HCC}$ has nano-scale water and oil components, and therefore it is expected that HCC would also be an effective additive in

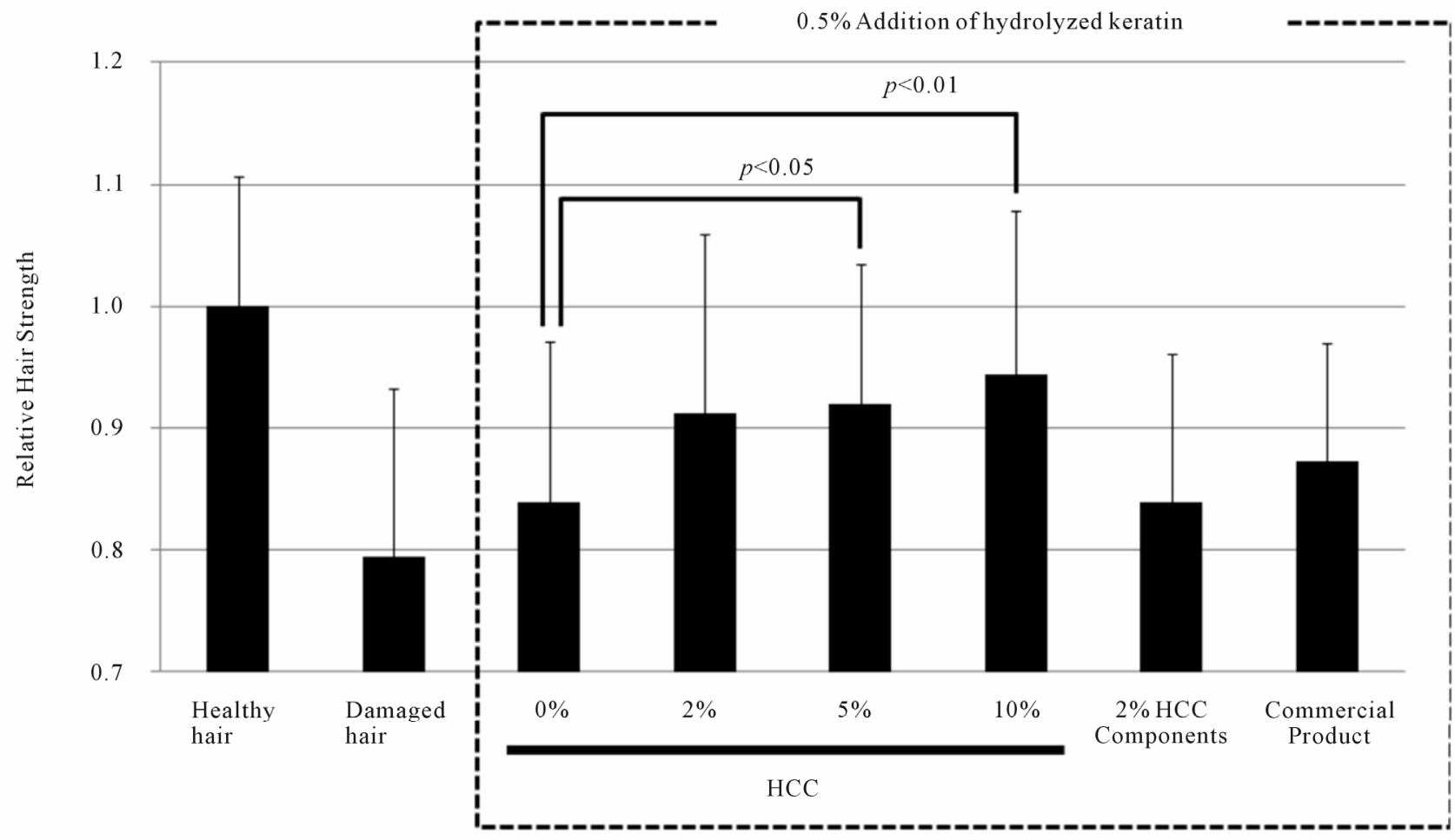

Figure 5. Relative strength of hair as determined by the amount of weight necessary to break a single strand of hair. 
products that modify the surface of the hair by coating it with a mixture of oil and water, which improves the feel to the touch of the hair.

To meet the needs of all the consumers, it is indeed important to develop various effective active ingredients; however, it is equally important to ensure that those ingredients can be delivered past the cuticle to the core of the hair. It is expected that demand for effective penetration-enhancing agents will continue to rise, and that there will be a wide variety of potential applications for HCC.

\section{Acknowledgements}

We would like to thank Miss Ariella Coler-Reilly who wrote the English translation of this paper.

\section{REFERENCES}

[1] Japan Hair Color Industry Association (JHCIA), "The Kinds of Hair Coloring Products," 2011. http://www.jhcia.org

[2] T. Ikeda, K. Yamada and M. Ono, "Functional Characteristics of the Raw Materials in Hair Care," Fragrance Journal, Vol. 35, No. 6, 2007, pp. 55-60.

[3] S. Nojima, J. Sunamoto and K. Inoue, "Liposome," Nankodo Co., Ltd., Tokyo, 1988.

[4] Y. Yamaguchi, "Treatment Utilizing DDS," In: H. Kuwano, Ed., Fundamentals and applications of MEMS/NEMS Engineering, Technosystem Co., Ltd., Tokyo, 2009, pp. 228-233.

[5] University of Tokyo Center for NanoBio Integration, "NanoBio Future Seen from Interdisciplinary Research in Pharmaceutical Science and Technology," X-Knowledge Co., Ltd., Tokyo, 2010.

[6] Y. Yamaguchi and R. Igarashi, "DDS to Support Skin Regeneration and Percutaneous Absorption: Development of Biomimetic NANOCUBE SYSTEM," Biotechnology Journal, Vol. 6, No. 5, 2006, pp. 585-589.

[7] M. Musashi and Y. Yamaguchi, "Development of Cosmetic Formulations 'NANOCUBE' Encourages Skin Regen- eration by the Self-Healing Ability," COSMETICSTAGE, Vol. 1, No. 7, 2007, pp. 56-60.

[8] Y. Yamaguchi, "Development of Topical Base for Promoting Transdermal Delivery. Effective Use of the Intercellular Lipid Present in the Outermost Layer of the Skin," Drug Delivery Using Functional DDS Carriers, CMC Publishing Co., Ltd., Tokyo, 2006, pp. 306-312.

[9] Y. Yamaguchi, "Research Trends of Trandermal Delivery in the Field of Cosmetics," Percutaneous Absorption in Aromatherapy, Aromatopia, Vol. 17, No. 6, 2008, pp. 15 .

[10] Y. Yamaguchi, Y. Suzuki, T. Nagasawa, Y. Kubota and M. Isogai, "Pigmentation and Improve Wrinkles by Multifunctional Soft Matter. New Concept for Trandermal Delivery System," Fragrance Journal, Vol. 37, No. 5, 2009, pp. 97-100.

[11] M. Hosokawa, "Damage by the Hair Perm, Coloring, Sun, Heat and Mechanical Force," In: Hair Scientists Association, Ed., Latest Hair Science, Fragrance Journal Ltd., Tokyo, 2003, pp. 220-259.

[12] A. K. Allen, J. Ellis and D. E. Rivett, "The Presence of Glycoproteins in the Cell Membrane Complex of a Variety of Keratin Fibres," Biochimica et Biophysica Acta, Vol. 1074, No. 2, 1991, pp. 331-333. doi:10.1016/0304-4165(91)90172-D

[13] A. Kanaya, R. Maruyama, K. Fujii and K. Kanayama, "Hair Repair Agent," Japan Patent No. 267844, 2003.

[14] M. Yoshioka, H. Araya, K. Adachi and S. Uchida, "Hair Cosmetic," Japan Patent No. 247692, 2005.

[15] Y. Tuda, "Effectiveness as Hair Care Ingredient Derived from the Hydrolysis of Waterfowl Feather Keratin," Fragrance Journal, Vol. 38, No. 7, 2010, pp. 74-77.

[16] Y. Yamashita, T. Sato, K. Sakamoto and Y. Yamaguchi "Shear-Induced Release Model of Solubilized Oil in Diluted Liquid Crystal Solution," Proceedings of International Association of Colloid and Interface Scientists Conference, Sendai, 13-18 May 2012. 